\title{
Evaluación de la calidad lagrimal en el Perro Sin Pelo del Perú mediante la técnica de Tiempo de Ruptura de la Película Lagrimal Precorneal
}

\author{
Evaluation of tear quality of peruvian hairless dog through the use of the tear film break-up time. \\ Joanna Flores ${ }^{1}$, Elizabeth Hinostroza ${ }^{1}$, Ricardo Grandez ${ }^{1}$, Francisco Canales ${ }^{2}$, Enrique \\ Serrano-Martínez ${ }^{1}$
}

\section{RESUMEN}

Objetivo: el presente estudio tuvo por objetivo evaluar la calidad lagrimal en el perro sin pelo del Perú mediante el uso de la prueba de tiempo de Ruptura de la película lagrimal precorneal (TRPLP). Material y métodos: El estudio se realizó en la ciudad de Lima entre los meses de Julio a Diciembre del año 2008. Se evaluaron 125 canes de diferentes edades, tamaños y de ambos sexos. Resultados: En los resultados de la prueba de TRPLP se observó un alto porcentaje de perros con mala calidad lagrimal: 94,4\% (118/125) en el ojo derecho y el 92\% (115/125) en el ojo izquierdo. No se encontró asociación entre el sexo, estrato etáreo y lado del ojo evaluado para la prueba de (TRPLP). Conclusión: Se concluye que existe un porcentaje elevado de perros sin pelo del Perú con una mala Calidad lagrimal.

PALABRAS CLAVE: Oftalmología canina, perro sin pelo del Perú, calidad lagrimal, prueba de tiempo de ruptura de la película lagrimal precorneal.

\section{SUMMARY}

Objectives: The purpose of this study was to evaluate the tear quality of Peruvian hairless dog through the use of the tear film break up time (TFBUT). Material and methods: The study took place in Lima Between July 2008 and December 2008. Results: A total of 125 animals of different of poor quality: $94.4 \%(118 / 125)$ in the rigth eye and $92 \%(115 / 125)$ in the left eye and without significant difference due to sex, age and eye side evaluated. Conclusion: It is concluded that a high percentage of peruvian hairless dog a poor tear film quality.

KEYWORDS: Canine ophthalmology, peru hairless dog, quality tear production, tear film break up time.

\section{INTRODUCCIÓN}

El Perro sin Pelo del Perú (PSPP) es la única raza registrada en la Federación Cinológica Internacional (FCI) como raza autóctona del Perú (1). Las características más saltantes son la ausencia total o parcial de pelo y de dientes premolares, debido a la presencia de un genotipo determinado por un rasgo dominante autosomal denominado displasia ectodermal, que a su vez condiciona la falta de pestañas $(2,3)$ y de glándulas de Meibomio que son glándulas sebáceas modificadas localizadas a lo largo de todos los márgenes palpebrales, responsables de la producción de la capa lipídica que estabiliza la película lagrimal y reduce la pérdida de ésta por evaporación (4). La displasia ectodermal también puede 
condicionar la presentación de alacrima congénito pero el mecanismo de desarrollo de esta alteración no está claro, algunas teorías la relacionan con aplasia del núcleo salivar superior, disfunción del sistema nervioso periférico o central e hipoplasia o aplasia de la glándula lagrimal. Existe una fuerte predisposición racial para alacrima congénita y por tanto a la presentación de queratoconjuntivis seca (QCS) en la raza Yorkshire Terrier donde el número de perros de un estudio permitió establecer esta predisposición, también se sospecha que perros como el Crestado Chino, que tiene características muy semejantes al PSPP, pueden padecer de esta alteración congénita, sin embargo debido a la falta de un ejemplar de esta raza con esta patología no se ha establecido una significancia estadística (5).

Mediante la prueba de lágrimas de Schirmer (STT) (6) se determinó que el 7,9\% (101/8) de canes de esta raza presentan QCS y el 8,9\% (101/9) son sospechosos para dicha patología.

La película lagrimal precorneal (PLP) es una cubierta compleja de fluido trilaminar, que consiste de una capa interna mucínica; una capa intermedia acuosa, más gruesa; y una capa lipídica externa relativamente delgada $(7,8)$. La capa mucínica está compuesta por sialoglicoproteínas que tiene una porción lipofílica que permite la conexión entre ella y la córnea, y una porción hidrofílica que permite una estrecha conexión con la capa acuosa (9). La mucina es producida por las células caliciformes de la conjuntiva. La porción acuosa de la PLP es producida conjuntamente por la glándula lagrimal orbitaria y la glándula nictitante. (10-12). La porción lipídica es producida por las glándulas de Meibomio que producen lípidos como colesterol y algunos triglicéridos modificados, los cuales son específicos en cada especie e incluso en cada individuo; estas glándulas se encuentran en mayor número en el párpado y vacía su contenido cuando son suavemente comprimidas durante el proceso de parpadeo. Su función es retardar la evaporación y estabilizar la superficie lagrimal $(7,13)$.

La composición de la lágrima es de $99 \%$ de agua, además de una enzima llamada lisozima; así como vitaminas, sales inorgánicas, glucosa, y otros; componentes específicos de cada especie y de cada glándula lagrimal $(7,13)$ y cubren las necesidades metabólicas de las capas superficiales de la córnea avascular, también participa en retirar todos lo metabolitos tóxicos como ácido láctico y dióxido de carbono; así como detritus celulares y bacterias $(7,12)$.
La ausencia o desequilibrio de estos componentes va a causar un efecto de raspado del párpado sobre la superficie ocular originando la Queratoconjuntivitis Seca (QCS) que es una enfermedad ocular muy común que se caracteriza por una disminución variable de la capa acuosa de la película lagrimal precorneal, y causa desecación e inflamación de la conjuntiva y córnea, así como dolor ocular, conjuntivitis, melanosis y vascularización corneal que pueden estar presentes dependiendo del estadío de la enfermedad, con presencia de descarga ocular mucoide que puede ser confundida con conjuntivitis bacteriana $(2,10,12)$. La QCS además de estar asociada a una deficiencia cuantitativa en el componente acuoso de las lágrimas también puede deberse a una deficiencia cualitativa de las capas de mucina o de lípidos de la PLP, que puede causar una inestabilidad de esta (14).

Las alteraciones de la PLP son el resultado de la interacción de un conjunto de factores con signología de QCS. En los perros, la QCS se caracteriza por ser un desorden inmunomediado asociado a condiciones autoinmunes sistémicas, otras causas incluyen enfermedad infecciosa, como distemper, intoxicación asociado a sulfonamidas u otras drogas, remoción quirúrgica de la glándula del tercer párpado, trauma facial y alacrima congénita (2).

Los hallazgos clínicos de los pacientes con QCS de tipo cualitativa incluyen la presencia de queratoconjuntivitis crónica, ulceración corneal, con una producción cuantitativa normal $(15-25 \mathrm{~mm} /$ min) de lágrimas, sin acompañarse de otros factores de irritación. Algunos casos se caracterizan por la ausencia de secreción ocular mucosa, que se relaciona directamente con la falta de mucina, observándose cantidades muy disminuidas de células caliciformes hasta su ausencia total (8). En la QCS de tipo cuantitativa se observan signos como descarga ocular mucopurulenta, diferentes grados de conjuntitivis, queratitis pigmentaria, ulceración corneal y ceguera (5).

El diagnóstico de la QCS cuantitativa se basa en la historia clínica, signos clínicos y resultados de la prueba de Schirmer (STT) menor a $10 \mathrm{~mm}$ de producción lagrimal durante un minuto; la QCS cualitativa lipodeficiente se basa en la presencia de signos clínicos, adicionalmente se cuenta con STT normal o aumentado, el tiempo de ruptura de la película lagrimal precorneal (TRPLP) anormal y Rosa de Bengala negativo, mientras que en la QCS cualitativa mucinodeficiente se observa un ojo con 
STT normal o aumentado, TRPLP anormal y un Rosa de Bengala positivo $(12,15)$.

Este diagnóstico de la QCS cualitativa debe ser confirmado con una biopsia conjuntival que permita cuantificar el número de células caliciformes $(5,16,17)$. Aunque en la mayoría de los pacientes existe una disminución en la producción de lágrimas, algunos cambios incipientes en la superficie ocular son debidos a deficiencias cualitativas de la PLP, sin existir cambios cuantitativos de la misma (18).

El presente estudio busca evaluar la calidad lagrimal en el Perro sin Pelo del Perú mediante el uso de la prueba del Tiempo de Ruptura de la Película Lagrimal Precorneal.

\section{MATERIAL Y MÉTODOS}

\section{Lugar de тиеstreo}

El estudio se realizó en la ciudad de Lima en los distritos de Chaclacayo, La Molina, Surco y Ate Vitarte, entre los meses de julio a diciembre del año 2008.

\section{Animales y tamaño muestral}

El tamaño muestral fue de 120 animales, número que se obtuvo utilizando la fórmula de comprobación de una proporción aplicada a poblaciones finitas (19); y se utilizó como proporción referencial el valor de 7,9\%, valor obtenido referido a QCS mediante la prueba de STT (6). El número de animales considerados en el estudio fue de 125 ejemplares de la raza PSPP. Los criterios de inclusión para el estudio fueron: animales mayores de 3 meses de edad, sin enfermedades detectables al examen clínico y sin antecedentes de enfermedad en al menos 3 meses antes del inicio del estudio. Se excluyeron del estudio a perros muy nerviosos o agresivos.

Se clasificaron a los animales del estudio de acuerdo al sexo: hembras y machos; por edad: cachorros, $\leq$ a 1 año; adultos, > a 1 año y $\leq$ a 7 años; y gerontes, > a 7 años. Según tamaño: pequeños, de 25 a $40 \mathrm{~cm}$; medianos, de 41 a $50 \mathrm{~cm}$; grandes, de 51 a $65 \mathrm{~cm}$, a la cruz según la FCI.

\section{Metodología}

Se montó un módulo de trabajo en ferias y concursos donde se convocaron a los animales de esta raza. También se realizaron muestreos en criaderos y crianzas particulares.

Con conocimiento de los propietarios sobre el objetivo y los procedimientos a realizar en el estudio, se realizó una encuesta para la identificación de los animales.

\section{Examen Oftalmológico}

El examen oftalmológico consistió en un examen clínico general externo del ojo que consistió en la evaluación externa de la córnea, pestañas, glándula nictitante, conjuntiva, conducto nasolagrimal. Luego se realizó el examen de medición de lágrimas con las tiras de Schirmer. Los resultados de 15 a $25 \mathrm{~mm}$ fueron considerados como normales, mayores a $25 \mathrm{~mm}$ epífora, de 10 a $14 \mathrm{~mm}$ como sospechosos y menores a $10 \mathrm{~mm}$ como una producción lagrimal anormal $(12,18)$.

\section{Prueba de Tiempo de Ruptura de la Película Lagrimal Precorneal (TRPLP)}

Para realizar esta prueba se colocó una gota de fluoresceína en el ojo y se dejó que el paciente pestañee o se le juntaron los párpados manualmente para poder lograr en todo el globo ocular una película lagrimal delgada con la tinción. Luego se mantuvo el ojo abierto por unos segundos, se puso la luz de cobalto sobre la córnea y se observó en qué momento se evaporó la película de fluoresceína. Cuando esto ocurrió se vieron manchas secas (oscuras), que indicaban el TRPLP; este procedimiento se repitió en el otro ojo. Los valores mayores o iguales a 15 segundos se consideraron normales, si fue menor de 15 segundos fue anormal; los valores obtenidos entre 14 a 5 segundos se consideraron como predispuesto a presentar signos QCS o sospechoso a QCS; si fue menor de 5 segundos se considera positivo a QCS cualitativa $(12,15)$. Ambos métodos diagnósticos fueron realizados por una sola persona para reducir el margen de error en las mediciones.

\section{Análisis de datos}

Se determinaron los valores de TRPLP y adicionalmente se consideraron los valores de STT según ojo del animal para el total de los ejemplares estudiados. También se determinó la distribución de estos resultados según grupo etáreo, tamaño y sexo. La asociación entre estas variables independientes y los resultados para cada prueba diagnóstica fueron analizadas mediante el estadístico de Chi cuadrado. 


\section{RESULTADOS Y DISCUSIÓN}

El valor de la prueba de TRPLP nos indica una alta presentación de QCS cualitativa en esta raza, aunque en nuestro estudio no podemos definir si las causas de esta alteración se deben a una deficiencia de la capa lipídica o mucínica. Un estudio en pacientes humanos ha comprobado que una disminución en el TRPLP está más asociado con un ojo seco debido a deficiencia de mucina, (17), en un futuro, la mucina podría ser la clave en el tratamiento o la prevención de las infecciones microbianas de la superficie ocular y del ojo seco (20). Es necesario realizar una biopsia conjuntival para confirmar la deficiencia de una de estas capas (15-17).

En nuestro estudio se pudo observar un alto porcentaje de sospechosos a QCS cualitativa: 94,4\% (118/125) en el ojo derecho y el 92\% (115/125) en el ojo izquierdo (Tabla 1). En cuanto a los resultados de STT se observó un elevado porcentaje de animales

Tabla 1. Distribución proporcional de los casos de QCS según TRPLP.

\begin{tabular}{lllll}
\hline \multirow{2}{*}{ Diagnóstico } & \multicolumn{2}{c}{ Ojo derecho } & \multicolumn{2}{c}{ Ojo Izquierdo } \\
\cline { 2 - 5 } & $\mathbf{N}^{\mathbf{0}}$ & $\mathbf{\%}$ & $\mathbf{N}^{\mathbf{0}}$ & $\mathbf{\%}$ \\
\hline Normal & 2 & 1,6 & 2 & 1,6 \\
Sospechoso & 118 & 94,4 & 115 & 92 \\
Anormal & 5 & 4 & 8 & 6,4 \\
TOTAL & 125 & 100 & 125 & 100 \\
\hline
\end{tabular}

Tabla 2. Distribución proporcional de los casos de QCS según STT.

\begin{tabular}{lllll}
\hline \multirow{2}{*}{ Diagnóstico } & \multicolumn{2}{l}{ Ojo derecho } & \multicolumn{2}{c}{ Ojo Izquierdo } \\
\cline { 2 - 5 } & $\mathbf{N}^{\mathbf{0}}$ & $\mathbf{\%}$ & $\mathbf{N}^{\mathbf{0}}$ & $\mathbf{\%}$ \\
\hline Epífora & 22 & 17,6 & 25 & 20 \\
Normal & 90 & 72 & 91 & 72,8 \\
Sospechoso & 3 & 2,4 & 4 & 3,2 \\
Anormal & 10 & 8 & 5 & 4 \\
TOTAL & 125 & 100 & 125 & 100 \\
\hline
\end{tabular}

con epifora: $17,6 \%(22 / 125)$ en el ojo derecho y $20 \%$ (25/125)enelojoizquierdo (Tabla2). Estoposiblemente debido a las características de alopecia congénita que presenta este perro, sospechamos que existe una relación de nuestros resultados con la alacrima que puede padecer debido a la displasia ectodermal (3), que condiciona la ausencia de pestañas y por tanto falta de glándulas de Meibomio responsable de la formación de la capa lipídica que estabiliza la película lagrimal y reduce la pérdida de ésta por evaporación (4). Se ha demostrado que los síntomas de la QCS se exacerban por irregularidades en las proporciones de fosfolípidos en la secreción meiobiana. Al respecto, en un estudio, se ha encontrado una baja proporción de fosfolípidos neutrales y aniónicos en las glándulas de Meibomio de los pacientes con esta alteración en comparación con sujetos normales. No se sabe exactamente qué componente de los lípidos incrementa la estabilidad de la lágrima, algunos investigadores mencionan la importancia de los grupos hidroxilo como los que existen en la esfingomielina porque incrementan la unión de los puentes de hidrógeno de los lípidos. Otros autores, indican que algunos fosfolípidos neutrales disminuyen la tensión superficial y por tanto mejora la estabilidad de la capa (21). Debemos obtener estos datos en el PSPP para determinar con exactitud la causa de la QCS tipo cualitativa.

El STT mide el incremento del reflejo de la secreción de la porción acuosa de las lágrimas, que son producidas por la glándula lagrimal del tercer párpado en el perro (20). En los resultados de la prueba de STT apreciamos el alto porcentaje de perros con epífora, como causas principales de esta alteración se mencionan a la presencia de párpados anormales, cilios ectópicos, puncta lagrimal dañada, y desórdenes funcionales y mecánicos. En humanos y en perros braquicéfalos como el Shih Tzu, se describe que en las epiteliopatías corneales la capa de mucina no puede mantenerse, y por tanto más células superficiales corneales se separan, lo que impide el pasaje de las lágrimas hacia el sistema nasolacrimal causando una

Tabla 3. Distribución proporcional de los casos de QCS según tamaño diagnosticada por TRPLP.

\begin{tabular}{llllllllll}
\hline \multirow{2}{*}{ Lado del Ojo } & $\begin{array}{l}\text { Diagnóstico } \\
\text { TRPLP }\end{array}$ & \multicolumn{2}{l}{ Pequeño } & \multicolumn{2}{c}{ Mediano } & \multicolumn{2}{c}{ Grande } & \multicolumn{2}{c}{ Total } \\
\cline { 2 - 9 } & N & 2 & 4.2 & 0 & 0 & 0 & 0 & 2 & 1,6 \\
\multirow{3}{*}{ Derecho } & Normal & 44 & 91,7 & 54 & 98,2 & 20 & 90,9 & 118 & 94,4 \\
& Sospechoso & 2 & 4,2 & 1 & 1,8 & 2 & 9,1 & 5 & 4,0 \\
& Anormal & 2 & 4,2 & 0 & 0 & 0 & 0 & 2 & 1,6 \\
\multirow{3}{*}{ Izquierdo } & Normal & 41 & 85,4 & 54 & 98,2 & 20 & 90,9 & 115 & 92,0 \\
& Sospechoso & 5 & 10,4 & 1 & 1,8 & 2 & 9,1 & 8 & 6,4 \\
\hline
\end{tabular}


Tabla 4. Distribución proporcional de los casos de QCS según Prueba de Shrimer.

\begin{tabular}{lllllllll}
\hline Diagnóstico & \multicolumn{7}{l}{ Ojo derecho } & \multicolumn{7}{l}{ Ojo Izquierdo } \\
\cline { 2 - 9 } & \multicolumn{2}{l}{ Prueba STT } & \multicolumn{2}{c}{ Prueba TRPLP } & \multicolumn{2}{c}{ Prueba STT } & \multicolumn{2}{c}{ Prueba TRPLP } \\
& $\mathrm{N}$ & $\%$ & $\mathrm{~N}$ & $\%$ & $\mathrm{~N}$ & $\%$ & $\mathrm{~N}$ & $\%$ \\
\hline Epifora & 22 & 17,6 & 0 & 0 & 25 & 20 & 0 & 0 \\
Normal & 90 & 72 & 2 & 1,6 & 91 & 72,8 & 2 & 1,6 \\
Sospechoso & 3 & 2,4 & 118 & 94,4 & 4 & 3,2 & 115 & 92 \\
Anormal & 10 & 8 & 5 & 4 & 5 & 4 & 8 & 6.4 \\
Total & 125 & 100 & 125 & 100 & 125 & 100 & 125 & 100 \\
\hline
\end{tabular}

epífora (22). En nuestro estudio, realizamos una evaluación previa de los párpados, cilios ectópicos, y ducto nasolagrimal, por tanto sospechamos que la causa de epifora puede deberse a la inestabilidad lagrimal causada por falla de la capa de mucina o de la capa lipídica ya que esta última contiene mucina soluble que disminuye la tensión superficial y mejora la extensión de la capa acuosa haciéndola más viscosa. Las pruebas de STT y TRPLP son complementarias porque cada prueba mide porciones diferentes de la PLP. Además, estudios sobre la relación que existe entre las pruebas para medir la PLP y pacientes con la enfermedad de ojo seco han demostrado que el STT está asociado con el TRPLP (17).

En la evaluación estadística no se encontró asociación entre sexo, estrato etáreo y lado de ojo evaluado para la prueba de TRPLP. La evaluación de la correlación entre los resultados de las pruebas STT y TRPLP fue bajo, tanto en el ojo izquierdo $(\mathrm{r}=0,34$, $\mathrm{p}=0,012)$ como en el derecho $(\mathrm{r}=0,37, \mathrm{p}=0,002)$. Asimismo, se observó un porcentaje mayor de ojos izquierdos anormales para TRPLP en animales pequeños y un porcentaje mayor de ojos derechos anormales para STT, estas diferencias estadísticas no tienen trascendencia biológica. Los detalles de estos resultados se presentan en las tablas 3 y 4 , respectivamente.

\section{CONCLUSIONES}

Los perros sin pelo del perú tienen una deficiencia cualitativa en la producción lagrimal.

Es necesario realizar otras pruebas diagnósticas como una biopsia conjuntival para saber si el origen de la QCS cualitativa es debido a una deficiencia de mucina o de lípidos.

\section{Correspondencia}

Elizabeth Hinostroza Meza

Correo electrónico: clarisa.hinostroza@upch.pe

\section{REFERENCIAS BIBLIOGRAFICAS}

1. Maniero E. Introducción a la morfología. Lima, Perú: Universidad Alas Peruanas; 2004. p. 199 - 232.

2. Herrera H, Weichsler N, Rodriguez J, García de Jalón J. Severe unilateral unresponsive Keratoconjunctivitis sicca in 16 juvenile Yorkshire Terrier. Vet Ophthalmol. 2007; 10:285-288.

3. Mecklenburg L. An overview on congenital alopecia in domestic animals. European Soc of Vet Dermatol. 2006;17:393-410.

4. Hartley C, Williams D, Adams V. Effect of age, gender, weight, and time of day on tear production in normal dogs. Vet Ophtalmol. 2006; 9: 53-57.

5. Westermeyer H, Ward D, Abrams K.. Breed predisposition to congenital alacrimia in dogs. Vet Ophthalmol. 2009; 12:1-5.

6. Aparcana Y. Prevalencia de queratoconjuntivitis seca en el perro sin pelo del Perú. Tesis para obtener grado de Médico Veterinario Zootecnista. Lima, Perú: Universidad Peruana Cayetano Heredia, 2007. 18pp.

7. Moore C. Qualitative tear film disease. Vet Clin North Am. 1990; 20: 565-581.

8. Kinoshita S, Kiorpes T, Friend J, Thoft R. Goblet cell density in ocular surface disease. A better indicator than tear mucin. Arch Ophthlmol. 1983; 101: 12481287.

9. Corfield A, Donapaty S, Carrington S, Hicks S, Schauer R, Kohla G. Identification of 9-O-acetyl$\mathrm{N}$-acetylneuraminic acid in normal canine preocular tear film secreted mucins and its depletion in Keratoconjunctivitis sicca. Glycoconjugate Journal. 2005; 22: 409-416.

10. Withely R, Mc Laughin S, Gilder B, Lindley D. The treatment for keratoconjuntivitis sicca. Vet Med. 1991; 3:1076-1093.

11. Peiffer R, Simon M. Enfermedad cualitativa de la película lagrimal: Oftalmología de pequeños animales. 3ra edición. Madrid, España. Elsevier; 2002.p. 219-254.

12. Tardón R. Queratoconjuntivitis seca cuantitativa y cualitativa (Internet). Primer Congreso Virtual Veterinarios en Web; 2008. (Citado el 19 enero del 2009). Disponible en: http://www.veterinariosenweb. 
com/v08/index.php

13. Herrera D. Canine keratoconjunctivitis sicca. Mexico DF, Mexico: SAVA Congress /World Small Animal Veterinary Association; 2005. (Citado el 17 marzo del 2009). Disponible en: http://www.ivis.org/ proceedings/wsava/2005/105.pdf

14. Berdoulay A, English R, Nadelstein B. Effect of topical $0.02 \%$ tacrolimus aqueus suspension on tear production in dogs with keratoconjunctivitis sicca. Vet Ophthalmol. 2005; 8: 225-232.

15. García G, Avidan Y, Calderon J, Whitley R. Patologías oftalmológicas asociadas a alteraciones de los componentes de la película precorneal. Ciencia Veterinaria (Internet). 1994; 6 (Citado el 17 marzo del 2009). Disponible en: http://www.fmvz.unam. $\mathrm{mx} / \mathrm{fmvz} /$ cienciavet/revistas/CVvol6/CVv6c8.pdf

16. Moore C, Wilsman N, Nordheim E, Majors L, Collier L. Density and distribution of canine conjuntival goblet cells. Invest Ophthalmol Vis Sci. 1987; 28:1925-1932.

17. Nichols K, Nichols J, Mitchell G. The relation between tear film tests in patients with dry eye disease. Ophthal Physiol Opt. 2003; 23:553-560.
18. Esteban J. Sistema lagrimal. Atlas de oftalmología del perro y gato. Zaragoza, España: Servet; 2007. p. 158-169.

19. Steel R, Torrie J. Bioestadística: Principios y procedimientos. 2a edición. Bogotá: McGraw-Hill; 1985.p. 622.

20. Davidson H, Kuonen V. The tear film and ocular mucins. Vet Ophtalmol. 2004; 7: 71-77.

21. Peters K, Millar T. The role of different phospholipids on tear break-up time using a model eye. Current Eye Research.2002; 25:55-60.

22. Saito A, Kotani T. Tear production in dogs with epiphora and corneal epitheliopathy. Vet Ophthalmol. 1999; 2:173-178.

Recibido: 01/08/2013

Aceptado: 21/11/2013 